\title{
Visualizing the interaction of Acanthamoeba castellanii with human retinal epithelial cells by spontaneous Raman and CARS imaging
}

\begin{tabular}{|c|c|}
\hline Journal: & Journal of Raman Spectroscopy \\
\hline Manuscript ID & JRS-17-0226.R1 \\
\hline Wiley - Manuscript type: & Research Article \\
\hline Date Submitted by the Author: & 28-Sep-2017 \\
\hline Complete List of Authors: & $\begin{array}{l}\text { Naemat, Abida; University of Nottingham, School of Physics and Astronomy } \\
\text { Sinjab, Faris; University of Nottingham, School of Physics and Astronomy } \\
\text { McDonald, Alison; University of Edinburgh, Institute of BioEngineering } \\
\text { Downes, Andrew; University of Edinburgh, Institute for Materials \& } \\
\text { Processes; } \\
\text { Elfick, Alistair; University of Edinburgh, Institute for Materials \& Processes } \\
\text { Elsheikha, Hany; University of Nottingham, School of Veterinary Medicine } \\
\text { and Science } \\
\text { Notingher, Ioan; University of Nottingham, School of Physics and } \\
\text { Astronomy }\end{array}$ \\
\hline Keywords: & $\begin{array}{l}\text { Acanthamoeba castellanii, human cells, host-pathogen interaction, Raman } \\
\text { micro-spectroscopy, coherent anti-stokes Raman spectroscopy }\end{array}$ \\
\hline
\end{tabular}




\title{
Visualizing the interaction of Acanthamoeba castellanii with human retinal epithelial cells by spontaneous Raman and CARS imaging
}

\author{
Abida Naemat, ${ }^{\mathrm{a}}$ Faris Sinjab, ${ }^{\mathrm{a}}$ Alison McDonald, ${ }^{\mathrm{b}}$ Andy Downes, ${ }^{\mathrm{b}}$ Alistair Elfick, ${ }^{\mathrm{b}}$ Hany M. \\ Elsheikha ${ }^{\mathrm{c}}$ and Ioan Notingher ${ }^{*}$,a \\ ${ }^{a}$ School of Physics and Astronomy, University of Nottingham, Nottingham, NG7 2RD, United \\ Kingdom \\ ${ }^{b}$ School of Engineering, Institute for BioEngineering, University of Edinburgh, The King's \\ Buildings, Mayfield Road, Edinburgh EH9 3DW, United Kingdom \\ ${ }^{c}$ School of Veterinary Medicine and Science, University of Nottingham, College Road, \\ Leicestershire, LE12 5RD, United Kingdom
}

*Ioan Notingher, E-mail: ioan.notingher@nottingham.ac.uk; Tel: +44 01159515172 


\begin{abstract}
Improved understanding of the mechanism of nutrient's uptake can enable targeted manipulation of nutrient sensing pathways in medically important pathogens to a greater degree than is currently possible. In this context, we present the use of spontaneous Raman micro-spectroscopy (RMS) and coherent anti-Stokes Raman spectroscopy (CARS) to visualise the time-dependent molecular interactions between the protozoan Acanthamoeba castellanii and host human cells. Human retinal pigment epithelial (ARPE-19) cells were prelabelled with deuterated Phe (L-Phe(D8)) and the uptake of the host derived L-Phe(D8) by $A$. castellanii trophozoites was measured by RMS for up to 48 hours post infection (hpi). This approach revealed a time-dependent uptake pattern of this essential amino acid by $A$. castellanii trophozoites during the first 24 hpi with complete enrichment with L-Phe(D8) detected in trophozoites at 48 hpi. In contrast, cell free $A$. castellanii trophozoites showed a modest uptake of only 16-18\% L-Phe(D8) from L-Phe(D8)-supplemented culture medium after 3h, 24h and 48h hpi. CARS microscopy was successfully used to monitor the reprogramming of lipids within the trophozoites as they engaged with host cells. The methodology presented here provides new advances in the ability to analyze the kinetic of amino acid acquisition by $A$. castellanii from host cell and extracellular environment, and to visualize lipid reprogramming within the trophozoite.
\end{abstract}

Key words: Acanthamoeba castellanii; Coherent anti-Stokes Raman scattering; Hostpathogen interaction; Isotope labelling; Raman Spectroscopy 


\section{Introduction}

Acanthamoeba species are single cell eukaryotic protozoa that can survive in a variety of environmental niches, including soil and water, ${ }^{1}$ swimming pools, ${ }^{2}$ eye wash stations, ${ }^{3}$ and noses and throats of asymptomatic individuals. ${ }^{4}$ Acanthamoeba exists in two different life cycle forms: trophozoite and cyst. Under favourable conditions, such as abundant food supply, appropriate temperature, osmolarity and neutral $\mathrm{pH}$, Acanthamoeba exists in the vegetative, infective and replicative trophozoite stage. In this form of the life cycle, the organism has a diameter of $10-25 \mu \mathrm{m}^{5}$ and exhibits irregular shape with locomotor pseudopods and surface acanthopodia, ${ }^{6}$ Under stress, the trophozoite stage transforms into a metabolically inactive cyst ${ }^{7}$ and encloses itself within a thick wall and becomes resistant to biocides, chlorination and antibiotics. ${ }^{8}$ Cystic stage transforms back to the trophozoite form with improved conditions in a process known as excystment. Although considered as freeliving ubiquitous protozoans, several species of Acanthamoeba can cause severe infections in humans, mainly localized in immune privileged sites, such as the brain ${ }^{8}$ and the eyes. ${ }^{9}$

Despite the considerable efforts to elucidate the pathogenesis of diseases caused by Acanthamoeba castellanii infection, there are still many gaps in our understanding of the mechanisms that drive the interaction between this organism and host cells. One such mechanism that significantly impacts the parasite pathogenicity is the pathways by which $A$. castellanii trophozoites access nutrients from their host cells in infected tissues. It is a known fact that A. castellanii trophozoites display striking differences in their metabolic functions once they become colonized by bacterial as compared to non-colonized trophozoites. For examples, increase in the concentration of free amino acids in the cytosol of $A$. castellanii was attributed to activation of the amoebal proteasome by Legionella pneumophila AnkB protein. ${ }^{10}$ Also, there is evidence that amino acids auxotrophy of L. pneumophila and its amoebic host was synchronized during evolution. ${ }^{11}$ 
Recent data revealed an active shikimate pathway in A. castellanii and was proven essential for the synthesis of aromatic amino acids. ${ }^{12,13}$ Mass spectrometry-based ${ }^{13} \mathrm{C}$ isotopologue profiling of ${ }^{13} \mathrm{C}$-prelabeled A. castellanii trophozoites revealed that this parasite is capable of de novo biosynthesis of nine amino acids, namely Ala, Asp, Glu, Ser, Phe, Tyr, Pro, Gly, and Thr. On the other hand, Val, Leu, Ile, Lys, and His did not acquire any ${ }^{13} \mathrm{C}$ label, indicating that de novo synthesis from glucose was not detectable for these five amino acids. However, there appears to be no or little work experimentally testing this relationship in the context of $A$. castellanii interaction with mammalian host cells. Indeed, our understanding of this crucial process is still limited because of various experimental and conceptual challenges.

Spontaneous Raman micro-spectroscopy (RMS) provides detailed molecular analysis of individual cells with micrometric spatial resolution. ${ }^{14-18}$ With the exception of Plasmodium falciparum, ${ }^{19-21}$ reports of RMS studies of protozoan parasites have been limited. Selective scanning RMS imaging was used to detect Neospora caninum tachyzoites colonizing human brain microvascular-endothelial cells. ${ }^{22}$ RMS time-course studies over 48 hours were used to determine the molecular changes in the apicomplexan protozoan parasite Toxoplasma gondii and in their surrogate human retinal pigment epithelial (ARPE-19) cells. ${ }^{23}$ More recently, stable isotope labelling of the aromatic amino acid phenylalanine (Phe) combined with RMS enabled real-time monitoring of the molecular exchange of Phe between $T$. gondii engaging with ARPE-19 cells, non-invasively. ${ }^{24}$ RMS has also been used with isotope labelling technology to differentiate the cellular components produced through distinct metabolic pathways, ${ }^{25-28}$ lipid uptake and metabolism inside human macrophages on a single cell level ${ }^{29}$ and proteome localization in single fission yeast cells. ${ }^{30}$ Furthermore, RMS was used to study the encystation and excystation process in Acanthamoeba polyphaga ${ }^{31}$ and to investigate the underpinning biochemical mechanism and the time frame for eradication of 
Acanthamoeba in ocular infections following exposure to polyhexamethylene biguanide (PHMB), a polymer used as a disinfectant and antiseptic for the treatment of Acanthamoeba keratitis. $^{32}$

Coherent anti-Stokes Raman scattering (CARS) microscopy is a complementary and closely related technique to spontaneous Raman spectroscopy which allows fast highresolution 3D imaging of cells. ${ }^{33-35}$ CARS has been successfully used for various applications in the life sciences, particularly in lipid biology, from imaging lipid membranes $^{33}$, to endogenous cellular lipid droplet distributions ${ }^{33,36}$ and even whole-organism lipid imaging. ${ }^{37}$ The use of two excitation lasers with a frequency difference tuned to a particular vibrational band amplifies the Raman response by a nonlinear optical interaction, allowing much faster measurement of a sample at a single Raman band. The most common Raman band used to generate image contrast with CARS is the $\mathrm{CH}_{2}$ symmetric stretching band at $\sim 2845 \mathrm{~cm}^{-1}$, which is a reliable lipid biomarker.

In the present study, we utilized RMS-coupled with isotope labelling and CARS imaging to characterize the uptake of amino acid Phe by $A$. castellanii trophozoites from human ARPE-19 cells. Because the frequencies of the vibrational modes of Phe depend on the mass of the atoms, the substitution of hydrogen with deuterium decreases the wavenumber of the characteristic Raman bands. This makes it possible to discriminate between Phe molecules originating from the human cells and Acanthamoeba. With the use of high-resolution CARS, we also investigated whether the interaction of $A$. castellanii trophozoites with host cells triggers reprogramming of lipid contents within trophozoites.

\section{Experimental}

\section{Cell culture conditions}


As an immune-privileged and easily accessible organ, the eye constitutes a favorable target for A. castellanii. Therefore, human retinal pigment epithelial (ARPE-19; American Type Culture Collection, Manassas, VA) cell line, which represents the main constituent of the blood-retinal barrier was selected as a model to investigate the molecular changes that accompany $A$. castellanii infection to the ocular surface. ARPE-19 cells were used at passage 11 and were maintained in DMEM supplemented with 1\% penicillin/streptomycin and 5\% heat-inactivated fetal bovine serum (FBS). Cells were incubated in $5 \% \mathrm{CO}_{2}$ incubator at $37^{\circ} \mathrm{C}$ and were passaged twice weekly. Cell viability was determined by trypan blue exclusion assay and cells were used in the experiments when their viability is $>98 \%$.

\section{Parasite strain and growth conditions}

Acanthamoeba castellanii T4 genotype, originally obtained from the American Type Culture Collection (ATCC \#50492), was used in the study. Acanthamoeba castellanii trophozoites were grown in $\mathrm{T}-75 \mathrm{~cm}^{2}$ tissue culture flasks (Sarstedt, UK) in autoclaved axenic peptoneyeast-glucose $(\mathrm{PYG})$ medium comprising $0.75 \% \mathrm{w} / \mathrm{v}$ proteose peptone $\left(\right.$ Oxoid $^{\mathrm{TM}}$, ThermoFisher Scientific, UK), $0.75 \% \mathrm{w} / \mathrm{v}$ yeast extract, and $1.5 \% \mathrm{w} / \mathrm{v}$ glucose in distilled water. Flasks were maintained in a humidified standard air incubator at $25^{\circ} \mathrm{C}$. Every 3-5 days the growth PYG medium was removed from culture flasks and replaced with fresh medium. Under these conditions more than $90 \%$ of $A$. castellanii remained bound to the flask as trophozoites.

\section{Stable Isotope Labelling by Amino Acids (SILAC)}

The aim of this experiment was to determine the molecular exchange between host ARPE-19 cells and A. castellanii trophozoites. The ARPE-19 cells were cultured in a specially- 
formulated medium containing all amino acids in unlabelled form, but Phe, which was added with the desired isotope label. To minimize the background signal of the L-Phe, ARPE-19 cells were synchronized overnight in serum-deficient, Phe-free customized DMEM culture medium. The cells were subsequently incubated with SILAC DMEM medium supplemented with L-Phe(D8). The doubling time (DT) of ARPE-19 cells grown in vitro was determined daily for a period of 6 days via cell counting using automated cell counter (LUNA-IITM, Labtech International Ltd., East Sussex, UK). After complete substitution of L-Phe by LPhe(D8) in ARPE-19 cells (defined as $\mathrm{I}_{960} /\left(\mathrm{I}_{960}+\mathrm{I}_{1004}\right)=1$, where $\mathrm{I}_{960}$ and $\mathrm{I}_{1004}$ are the intensities of Raman bands at $960 \mathrm{~cm}^{-1}$ and $1004 \mathrm{~cm}^{-1}$ ), the cells are challenged with $A$. castellanii trophozoites as described below.

\section{Infection of host cells with $A$. castellanii}

ARPE-19 cells $\left(10^{4}\right.$ cells/ml) labelled with L-Phe(D8) were seeded in titanium cell-chambers (sample holders), which were fitted in 6-well plastic cell culture plates, with a volume of $2 \mathrm{ml}$ DMEM/chamber. The sample holders were purpose-built to enable acquisition of Raman spectra of the cells and incorporated MgF2 coverslips ( $0.17 \mathrm{~mm}$ thick) at the bottom. Before introducing the parasites to ARPE-19 cells the culture medium was replaced with L-Phe-free media to avoid any false labelling of the parasite. Parasites were grown in PYG medium, but they were transferred to L-Phe-free DMEM media during host cell infection. The only source of L-Phe(D8) to the parasites came from the labelled host cells and L-Phe for host cells was from the parasites. Infection was initiated by adding A. castellanii trophozoites at a multiplicity of infection of 1 cell to 1 parasite. During Raman spectral mapping of live cells, A. castellanii trophozoites were found to move beyond the designated area of imaging before the completion of raster scan measurement at 0.5 seconds acquisition time. Thus, we had to 
perform the measurements on trophozoites fixed with $4 \%$ paraformaldehyde before starting the Raman spectral imaging.

\section{L-Ph(D8) uptake by isolated $A$. castellanii trophozoites}

We were also interested in testing the ability of isolated trophozoites to acquire L-Phe(D8) from the culture medium. To achieve this, we harvested $2 \times 10^{7} \cdot$ A. castellanii trophozoites from cultured flasks, followed by washing in $10 \mathrm{ml}$ of sterile Dulbecco's phosphate-buffered saline three times to remove any traces of PYG medium. The trophozoite pellet was then suspended in $2 \mathrm{ml}$ of $\mathrm{L}-\mathrm{Ph}(\mathrm{D} 8)$-supplemented medium and were subjected to Raman imaging for 24 hours.

\section{Spontaneous Raman micro-spectroscopy (RMS)}

Raman spectra were recorded using a purpose-built confocal Raman micro-spectrometer optimized for studying cell biology and host-parasite interaction. An inverted microscope (IX71, Olympus, Essex, UK) was used for the setup because it allows cell measurements without the limitation of the objective lens dipping in and contaminating the culture media as opposed to up-right microscopes. The laser is focused through a MgF2 coverslip at the bottom of specially designed sample holders. A $785 \mathrm{~nm} \sim 170 \mathrm{~mW}$ laser (before objective) (Ti:sapphire laser, spectra-physics) was used for excitation of Raman spectra. To maximize the spatial resolution and collection efficiency for the Raman spectra, a $60 \times / 1.20$ NA waterimmersion objective (Olympus) was used to focus the laser (beam diameter expanded to match the objective pupil, laser spot $\sim 700 \mathrm{~nm}$ on sample) on individual cells as well as for collection of Raman scattered photons. The Raman scattered light was collimated and then focused on a $50-\mathrm{mm}$ diameter optical fiber connected to a spectrometer equipped with a 830 lines per mm grating (spectral resolution of $1.5 \mathrm{~cm}^{-1}$ in the $600-1800 \mathrm{~cm}^{-1}$ region) and cooled 
deep-depletion back-illuminated CCD detector (Andor Technologies, Belfast, UK). Raman spectral imaging was performed by scanning areas of the trophozoites through the laser focus in a raster pattern ( $1 \mu \mathrm{m}$ step size) using a high-precision step-motor stage (Prior, Cambridge, UK) and acquiring Raman spectra at each position (1s per pixel). The spectrometer wavenumber axis was calibrated prior to each experiment using tylenol at an accuracy of 0.5 $\mathrm{cm}^{-1}$.

\section{CARS microscopy}

CARS images were acquired using a custom built multi-modal multiphoton microscope as described in Moura et al ${ }^{38}$. This system consists of a mode-locked ND:YVO4 laser source (PicoTrain, Spectra Physics) to generate the Stokes pulse (6ps, $1064 \mathrm{~nm})$ and also a 532nm output which is coupled into an optical parametric oscillator (OPO) providing tuneable excitation across 700-1000nm (Levante Emerald, APE). The two beams are coupled into an inverted microscope (Eclipse TE2000U, Nikon) with a Nikon BV 'C1' scanhead. Both beams were overlapped spatially and temporally on to the sample using a $25 \times / 1.05$ NA waterimmersion objective lens (Olympus). The CARS signal was acquired at the $2845 \mathrm{~cm}^{-1}$ Raman resonance by tuning the OPO to $817 \mathrm{~nm}$ wavelength. All images were acquired with $115 \mathrm{~mW}$ $(817 \mathrm{~nm})$ and $80 \mathrm{~mW}(1064 \mathrm{~nm})$ of excitation power at the sample plane.

\section{Fluorescence Staining}

Acridine orange (AO) staining was used to differentiate the morphological features of $A$. castellanii trophozoites from the host ARPE-19 cells. As a DNA intercalating dye, AO is used to differentially stain RNA and DNA inside eukaryotic cells. ARPE-19 Cells were fixed in $4 \%$ paraformaldehyde for $15 \mathrm{~min}$, followed by washing with 1x phosphate buffered saline (PBS) twice. Fixed cells were incubated with 5\% AO staining solution for 10 min. Imaging 
of AO-stained A. castellanii-infected ARPE-19 cells was performed, on the same cell, after completion of the Raman spectral imaging. Imaging was performed by using wide-field fluorescent microscope integrated on the confocal Raman micro-spectrometer. The retrospective positioning and identification of the cells was based on two thin marks engraved on the cell chambers (retro-positioning accuracy was $5 \mu \mathrm{m}$ ).

\section{Image analysis and data processing}

Data pre-processing and analysis was done by using in-house built functions in Matlab (The MathWorks, Natick, MA). The process of data pre-processing involved cosmic rays removal and background subtraction. The cosmic ray correction algorithm was design to detect the regions of the Raman spectra that had a gradient higher than the average gradient $\pm 10 \times$ the standard deviation. Once, detected, the algorithm removed 7 data points at the corresponding region and replaced them with values based on a linear extrapolated for that region. The background spectra were measured for each sample, and represented the mean of 100 Raman spectra measured as a $10 \mu \mathrm{m} \times 10 \mu \mathrm{m}$ raster scan in areas close to the cells of interest. Noise in the resulting data matrix was reduced by using singular value decomposition. The spectral images presented in Figures 1-6 were obtained by calculating the height of the specified Raman bands after subtraction of a local linear baseline for each particular band.

\section{Results}

\section{Uptake of L-Phe(D8) by A. castellanii from SILAC Media}

As a baseline, we first investigated whether $A$ castellanii can acquire L-Phe(D8) from the culture medium in the absence of host human cells. For this purpose, A. castellanii trophozoites were grown in normal medium conditions containing L-Phe and were 
subsequently incubated in media supplemented with L-Phe(D8). After 24 hours of incubation in the L-Phe(D8)-supplemented medium, Raman spectral imaging was performed. Raman bands were selected to map the distribution of lipids and proteins $\left(1449 \mathrm{~cm}^{-1}\right)$, nucleic acids (786 $\left.\mathrm{cm}^{-1}\right)$, L-Phe $\left(1004 \mathrm{~cm}^{-1}\right)$, and L-Phe(D8) $\left(960 \mathrm{~cm}^{-1}\right)$, as shown (Fig. 1). The results show that Raman spectra of A. castellanii consist of bands typically assigned to lipids, proteins and nucleic acids, similar to other cell types. ${ }^{14,15}$ The most intense bands can be assigned to molecular vibrations of lipid molecules, such as the $C=C$ stretching $1660 \mathrm{~cm}^{-1}$, $\mathrm{CH}_{2}$ deformation $1449 \mathrm{~cm}^{-1}, \mathrm{CH}_{2}$ twisting $1303 \mathrm{~cm}^{-1}$, $=\mathrm{C}-\mathrm{H}$ deformations $1260 \mathrm{~cm}^{-1}$, symmetric stretching of $\mathrm{N}\left(\mathrm{CH}_{3}\right)_{3}$ of choline group $719 \mathrm{~cm}^{-1}$. Raman bands corresponding to vibrational modes of the sugar-phosphate backbone in nucleic acids were identified at 788 $\mathrm{cm}^{-1}$ (O-P-O bonds) and $1098 \mathrm{~cm}^{-1}\left(\mathrm{PO}_{2}^{-}\right)$. The most intense Raman bands characteristic to proteins were observed at $\sim 1660 \mathrm{~cm}^{-1}$ and $1300-1400 \mathrm{~cm}^{-1}$, and were assigned to the Amide I and Amide III vibrations. $\mathrm{CH}_{2}$ vibrations of protein molecules also contributed to the 1445 $\mathrm{cm}^{-1}$ band. Of particular interest in this study is the ring-breathing mode of Phe, which was identified at $1004 \mathrm{~cm}^{-1}$ in native L-Phe. The wavenumber of this band is red-shifted to 960 $\mathrm{cm}^{-1}$ in L-Phe(D8) molecules. ${ }^{24,28}$ Thus, this was used in our study to analyze the uptake of LPhe by $A$. castellanii.

Figure 1 shows that the nucleus of $A$. castellanii can be identified as the region corresponding to the highest intensity in the Raman map corresponding to the $786 \mathrm{~cm}^{-1}$ band. The nucleus region was also characterised by a high concentration of proteins $\left(1004 \mathrm{~cm}^{-1}\right.$ and $1449 \mathrm{~cm}^{-1}$ maps). Regions of high concentration of lipids was also observed at locations (iii) and (iv) in the $1449 \mathrm{~cm}^{-1}$ map. At these locations, the $1303 \mathrm{~cm}^{-1}$ and $1660 \mathrm{~cm}^{-1}$ bands have high intensity. To provide an analysis of the overall uptake of L-Phe(D8) by A. castellanii from the culture medium, mean Raman spectra of individual A. castellanii were calculated by averaging all Raman spectra in the raster scan covering the area of the cell. Figure 2 presents 
spectral images of three typical $A$. castellanii and their mean Raman spectra. The uptake ratio of L-Phe(D8) was calculated by considering the intensity (height) of the L-Phe ( $\left.\mathrm{I}_{1004}\right)$ and LPhe(D8) ( $\left.I_{960}\right)$ bands by using the formula $I_{960} /\left(I_{1004}+I_{960}\right)$. This analysis showed that the concentration of L-Phe(D8) acquired by A. castellanii from the media in 24 hours represent $16-18 \%$ of the total amount of L-Phe in the trophozoites.

\section{Uptake of L-Phe(D8) by A. castellanii from ARPE-19 cells}

To investigate the exchange of L-Phe during the host-pathogen interaction, human ARPE-19 cells pre-labelled with L-Phe(D8) were inoculated with A. castellanii trophozoites. After 24 hpi, the interacting parasites and host cells were fixed in paraformaldehyde and measured by Raman raster scanning. Figure 3 presents typical Raman spectral maps corresponding to the native L-Phe $\left(1004 \mathrm{~cm}^{-1}\right)$ and the labelled L-Phe(D8) $\left(960 \mathrm{~cm}^{-1}\right)$, along with the maps corresponding to the $1449 \mathrm{~cm}^{-1}$ band.

The presence of the $960 \mathrm{~cm}^{-1}$ band and absence of the $1004 \mathrm{~cm}^{-1}$ band in the selected Raman spectra of the ARPE-19 cells indicate that human cells contain L-Phe(D8), but not LPhe. On the other hand, Figure 3 shows the presence of the $960 \mathrm{~cm}^{-1}$ band also in the Raman spectra of $A$. castellanii, an indication of the uptake of L-Phe(D8) from the ARPE-19 cells. It is also interesting to note that the Raman image corresponding to the $1449 \mathrm{~cm}-1$ band indicates a higher concentration of lipids at the junction between the $A$. castellanii and the ARPE-19 cell. However, the mean Raman spectra of $A$. castellanii in Figure 4 indicate that at this time point, the intensity of the $960 \mathrm{~cm}^{-1}$ band varied widely from cell to cell. A higher intensity of the $960 \mathrm{~cm}^{-1}$ band was associated with a decrease in the intensity of the L-Phe $1004 \mathrm{~cm}^{-1}$, such as in the case of samples 1 and 2 in Figure 4. The Raman spectra of some $A$. castellanii, e.g. Sample 3, indicated a complete saturation with L-Phe(D8) at 24 hpi.

Figure 5 presents Raman spectral maps of ARPE-19 cells infected with A. castellanii at 48 hpi. At this time point, the $1004 \mathrm{~cm}^{-1}$ Raman band corresponding to the native L-Phe can 
no longer be detected. The distribution of proteins appears homogenous (map corresponding to the $960 \mathrm{~cm}^{-1}$ band), while regions of high concentration of lipids can be identified at the periphery of the cell (1449 $\mathrm{cm}^{-1}$ map position iv). Intense bands assigned to nucleic acids (786 $\mathrm{cm}^{-1}$ band) can also be identified at position (iii), indicating the location of the nucleus.

The mean Raman spectra (Fig. 6) of $A$. castellanii trophozoites at $48 \mathrm{hpi}$ indicate that bands assigned to Phe were detected only at $960 \mathrm{~cm}^{-1}$. This result indicates that for all $A$. castellanii at 48 hpi, the native L-Phe was completely substituted with L-Phe(D8) uptaken from the host ARPE-19 cell.

\section{Dynamic of uptake of L-Phe(D8) at single parasite level}

The uptake of Phe by A. castellanii from the ARPE-19 host cells was expressed as the percentage of L-Phe(D8) from the total Phe, and was calculated as the ratio $\mathrm{I}_{960} /\left(\mathrm{I}_{960}+\mathrm{I}_{1004}\right)$. Figure 7a shows that compared to A. castellanii grown in L-Phe(D) media, for which $\mathrm{I}_{960} /\left(\mathrm{I}_{960}+\mathrm{I}_{1004}\right)=0.2$ at 24 hours, the $50 \%$ of the L-Phe in A. castellanii infecting ARPE-19 cells has been acquired from the human cells as L-Phe(D8) $\left(\mathrm{I}_{960} /\left(\mathrm{I}_{960}+\mathrm{I}_{1004}\right)=0.5\right)$. Nevertheless, the ratio showed large variations among cells (as also shown in Fig. 3), which is reflected in the large error bars in Fig. 7. At 48 hpi, complete substitution of L-Phe with LPhe(D8) was typically observed in all A. castellanii $\left(\left(\mathrm{I}_{960} /\left(\mathrm{I}_{960}+\mathrm{I}_{1004}\right)=1\right)\right.$.

The difference between the Raman spectra of $A$. castellanii infecting ARPE-19 cells and spectra of A. castellanii in medium containing L-Phe(D8) were calculated in order to investigate any changes related to L-Phe metabolism. In particular, we focused on whether LPhe(D8) was involved in the metabolism of tyrosine, by analyzing the $854 \mathrm{~cm}^{-1}$ and $834 \mathrm{~cm}^{-1}$ Fermi resonance doublet. This resonance is due to the interaction between the ring-breathing fundamental $\left(v_{1}\right)$ and the overtone of the nonplanar ring vibrations $\left(v_{16 a}\right) .{ }^{36}$ In the case of liquid p-cresol (doublet $842 \mathrm{~cm}^{-1}$ and $824 \mathrm{~cm}^{-1}, v_{16 a}$ at $413 \mathrm{~cm}^{-1}$ ), the substitution of $\mathrm{H}$ with D 
atoms removed the resonance because of the downshift of both vibrational modes, and typically only the $v_{1}$ band was observed in the Raman spectra: $824 \mathrm{~cm}^{-1}$ for p-cresol-2,6-D2 $\left(v_{16 \mathrm{a}}\right.$ at $\left.386 \mathrm{~cm}^{-1}\right)$ and $791 \mathrm{~cm}^{-1}$ for p-cresol-D4 $\left(v_{16 \mathrm{a}}\right.$ at $\left.362 \mathrm{~cm}^{-1}\right) .^{33}$ Based on these arguments, the presence of any deuterated tyrosine molecules following a potential metabolism of L-Phe(D8) by A. castellanii should make only the $v_{1}$ band detectable in the Raman spectra as a consequence of the removal of the Fermi resonance. In addition, the $v_{1}$ band is expected to downshift to the $854-790 \mathrm{~cm}^{-1}$ region. Nevertheless, the results in the difference spectra corresponding to A. castellanii and 48 hpi (Fig. 7b) indicate an increase in the $854 \mathrm{~cm}^{-1}$ band assigned to $v_{1}$, but no decrease in the intensity of the $834 \mathrm{~cm}^{-1}$ band to support the removal of the Fermi resonance. These spectral differences are consistent with changes in the ratio of intensities of the doublet, that may be related to the state of the hydroxyl groups in tyrosine, but show no evidence of the presence of deuterated tyrosine molecules in A. castellanii.

In order to obtain higher resolution visualization of the infection process, we utilized coherent anti-Stokes Raman scattering (CARS) microscopy for label-free observations of lipid structures in cells at different time-points after infection. Figure 8 shows example CARS images of $A$. castellani and ARPE-19 cells at $3 \mathrm{~h}, 24 \mathrm{~h}$ and $48 \mathrm{~h}$ post-infection. The highwavenumber region of the Raman spectra contained strong bands corresponding to the $\mathrm{C}-\mathrm{H}_{2}$ symmetric stretching at $2845 \mathrm{~cm}^{-1}$. The higher number of $\mathrm{C}-\mathrm{H}_{2}$ bond in lipid molecules compared to other common biomolecules, makes CARS a powerful technique for lipid imaging. Images were generated from maximum intensity z-projections of $3 \mathrm{D}$ imaging datasets (processed in ICY software ${ }^{39}$ ) which allows the total lipid content to be measured in the range from the coverslip surface to typically 5-20 $\mu \mathrm{m}$ above. Acanthamoeba trophozoites can be identified relatively easily with these 3D CARS datasets, as these cells typically do not spread out flat across the coverslip as were ARPE-19 cells. Also, the high spatial resolution 
allowed the vacuoles and concentrated structures within the cytoplasm/nucleus to be observed.

The CARS images also revealed some phenotypic changes at different time-points after infection. At 24 and 48 hpi, most of the Acanthamoeba trophozoites were rounded in shape, whilst several at the $3 \mathrm{hr}$ time-point were elongated, suggesting they were fixed as they were migrating across the host cell surface. Also, the concentration of lipid droplets within the trophozoites relative to the immediate surrounding appears to increase with time. The lipid droplet distribution in ARPE-19 cells at later time-points also appeared to be less uniform, with droplets appearing to collect close to the interface between them and any trophozoites in close proximity. This extensive lipid reprogramming suggests a coordinated response of the infected host cells as the trophozoites interact with them.

\section{Discussion}

We developed a methodology that combines Raman spectroscopy and stable isotope labelling to monitor molecular changes that occur during the interaction of the extracellular parasite $A$. castellanii with host ocular tissue using human ARPE-19 cells as a model. Our aim was to interrogate the amoeba's ability to acquire L-Phe(D8) from host cells. We also monitored the parasite's ability to uptake L-Phe(D8) while in isolation (i.e., growing freely in cell free L$\mathrm{Ph}(\mathrm{D} 8)$-supplemented medium). CARS imaging was also used for higher resolution structural imaging based on the Raman resonance at $2845 \mathrm{~cm}^{-1}$, particularly showing the distribution of lipid droplets within the trophozoites at different time points after infection. This combined approach allowed us to investigate the relative importance of metabolic exchange between $A$. castellanii and its host cells in vitro and provided information on amoeba-specific changes in chemical composition during interaction with ARPE-19 cells.

It was notable that extracellular $A$. castellanii trophozoites were not capable of acquiring 
L-Phe(D8) while growing in cell-free medium. Raman band related to isotope labelled LPhe(D8) at $960 \mathrm{~cm}^{-1}$ was very small even at 24 hours of incubation in L-Ph(D8)supplemented medium. On the other hand, when A. castellanii trophozoites were introduced to ARPE-19 cells, they showed fast uptake of L-Phe(D8), which was solely sourced from the host ARPE-19 cells as the medium used in the infection experiment did not contain any L$\mathrm{Ph}(\mathrm{D} 8)$. On average, A. castellanii trophozoites measured at 24 hpi showed uptake ratios between $40-60 \%$ of L-Phe (D8) from host cells. These results demonstrate that the uptake ratio of $\mathrm{L}-\mathrm{Ph}(\mathrm{D} 8)$ is enhanced by the physical contact between the host cell and the parasite. The importance of binding with host cells and protein trafficking in the initiation of $A$. castellanii infection has been demonstrated previously, showing that adhesion and secretion of a mannose-induced protein (MIP-133) by clinical A. castellanii isolate causes damage of corneal epithelial cells and that these cytopathic effects were mitigated by using chicken specific anti-MIP-133 antiserum. ${ }^{41}$

It is likely that parasites that are more quickly able to engage with host cells are those ones that are detected with complete substitution of L-Phe(D8) at 24 hpi. At 48 hpi, all the parasites were found completely enriched with L-Phe(D8). The clinical A. castellanii strain used in the present study is of the T4 genotype, which is known to bind more firmly to host target cells and leads to more cytotoxicity than other A. castellanii genotypes, ${ }^{42}$ which may explain the significant link between the T4 genotype with human corneal disease. These findings agree and contrast with results obtained in the apicomplexan protozoan parasite Toxoplasma gondii. While host cell free A. castellanii and T. gondii were less competent in acquiring L-Phe(D8) from culture medium, $T$. gondii tachyzoites were able to acquire LPhe(D8) from host cells very efficiently with uptake rates estimated as high as $10^{4}$ molecules/second. ${ }^{22}$ 
These findings reveal significant differences of amino acid uptake kinetics between these two different parasites, reflecting perhaps their different lifestyles. T. gondii is a strictly intracellular pathogen and has coevolved with its hosts in order to efficiently infect, replicate within, and be transmitted to new hosts to ensure survival and a continual infection cycle. Hence, dependence on host cells is fundamental for the survival of this pathogen. In contrast, A. castellanii is predominantly a free-living amoeba and causes disease under certain circumstances. Also, because amoeba organisms live under harsh conditions they developed a remarkable ability to adapt to numerous environmental factors (e.g., forming cysts) ${ }^{7}$ and to become less reliant on host to survive. Recent isotopologue profiling analysis showed that $A$. castellanii can synthesize de novo nine amino acids, namely Ala, Ser, Glu, Asp, Pro, Gly, Phe, Tyr, and Thr, whose precursors stem from degradation products of glucose via glycolysis, the PPP, and the TCA pathway, respectively. In contrast, the parasite cannot synthesize five AAs (Ile, Leu, Val, His, and Lys), ${ }^{12}$ indicating that the amoeba is auxotrophic for these amino acids. Earlier studies showed that Acanthamoeba minimal medium requires to be supplemented with Ile, Leu, Val, Arg, and Met, as long as glucose is present as a single carbon source, ${ }^{43}$ but Gly is needed for growth in minimal medium if acetate is the sole carbon source. Also, acetate was not found to be a suitable carbon/energy source for another Acanthamoeba species, A. culbertsoni ${ }^{44}$ Our results are interesting because despite the ability of $A$. castellanii trophozoites to synthesize aromatic amino acids de novo ${ }^{12}$ as they possess genes for the shikimate pathway, ${ }^{45}$ A. castellanii still acquires Phe efficiently from host cells. Acanthamoeba trophozoites appeared to have a higher lipid concentration at 24 and 48 hpi relative to the surrounding areas than at 3 hours, suggesting a subversion of host lipids. Acanthamoeba is rich in lipids; its plasma membrane consists of phospholipids (25\%), sterols (13\%), lipophosphonoglycan (29\%), and proteins (33\%). ${ }^{46,47}$ Phosphatidylcholine (45\%), phosphatidylethanolamine (33\%), phosphatidylserine (10\%), phosphoinositide (6\%), and 
diphosphatidylglycerol (4\%) constitute the major phospholipids in Acanthamoeba. The main fatty acids chains in this organism are oleic acids (40-50\%), and longer polyunsaturated fatty acids (20-30\%). ${ }^{48}$ Acanthamoeba also contains low levels of glycolipids and glucose accounts for about $60 \%$ of the sugars of the glycolipids of the whole cells and of the plasma membranes. Among sterols, the non-saponifiable fraction of the total lipids extracted from the trophozoites of pathogenic Acanthamoeba possesses ergosterol and 7dehydrostigmasterol. ${ }^{47}$ Therefore, lipids represent an essential source of energy storage, and can be expected to be targeted by Acanthamoeba during engagement with host cells for nutritional purposes and/or to modulate host-parasite interaction, including host inflammatory and immune responses. ${ }^{49}$

Taken together, these findings indicate that although $A$. castellanii trophozoites can exist in a free-living status, their interaction with host cells involves changes in their metabolic and nutritional needs. With further improvements in the capabilities and sensitivity of RMS, it might be possible in the future to study the molecular trafficking and turnover of multiple amino acids simultaneously, as well as isotope labelled lipids. Further research on the molecular basis of amino acid sensing in A. castellanii will benefit therapeutic development and ultimately enhance our success at combating this important pathogen.

\section{Acknowledgements}

This work was supported by Petplan Charitable Trust grant 11-38 (H. Elsheikha and I. Notingher). A. Naemat acknowledges the support from COMSATS Institute of Information Technology, Pakistan. The development of the Raman micro-spectrometer was supported by the Biotechnology and Biological Sciences Research Council UK research grant $\mathrm{BB} / \mathrm{G} 010285 / 1$. 


\section{References}

[1] J. K. Stehr-Green, T. M. Bailey and G. S. Visvesvara, Am. J. Ophthalmol. 1989 ; 107, 331.

[2] V. Muñoz and H. Reyes, Santiago de Chile Parasitol. Iatinoam. 2003 ; 58, 106.

[3] C. Paszko-Kolva and H. Yamamoto, Appl. Env. Microbiol. 1991 ; 57, 163.

[4] K. F. Van and H. Alizadeh, Invest. Ophthalmol. Vis. Sci. 1993 ; 34, 1937.

[5] R. J. Neff and R. H. Neff, Symp. Soc. Exp. Biol. 1969 ; 23, 51.

[6] B. Bowers and E. D. Korn, J. Cell Biol. 1968 ; 39, 95.

[7] B. Bowers and E. D. Korn, J. Cell Biol. 1969 ; 41, 786.

[8] F. Marciano-Cabral and G. Cabral, Clin. Microbiol. Rev. 2003 ; 16, 273.

[9] Di Cave, R. Monno, P. Bottalico, S. Guerriero, S. D’Amelio, C. D’Orazi, F. Berrilli, Eur. J. Clin. Microbiol. Infect. Dis. 2009 ; 28(6), 607.

[10]C. T. Price, T. Al-Quadan, M. Santic, I. Rosenshine and Y. Abu Kwaik, Science 2011 ; $334,1553$.

[11] C. T. Price, A. M. Richards, J. E. Von Dwingelo, H. A. Samara and Y. Abu Kwaik, Environ. Microbiol. $2014 ; 16,350$.

[12] E. Schunder, N. Gillmaier, E. Kutzner, W. Eisenreich, V. Herrmann, M. Lautner and K. Heuner, J. Biol Chem. $2014 ; 289,21040$.

[13] F. L. Henriquez, S. J. Campbell, B. K. Sundararaj, A. Cano A, S. P. Muench and C. W. Roberts, Protist 2015 ; 166, 93.

[14] D. Shipp, F. Sinjab, and I. Notingher, Adv. Opt. Photon. 2017 9; 315.

[15] B. Kann, H. L. Offerhaus, M. Windbergs and C. Otto, Adv. Drug Deliv. Rev. 2015 ; 89, 71. 
[16] M. Okada, N. I. Smith, A. F. Palonpon, H. Endo, S. Kawata, M. Sodeoka and K. Fujita, Proc. Natl. Acad. Sci. USA. $2012 ; 109,28$.

[17] F. C. Pascut, S. Kalra, V. George, N. Welch, C. Denning and I. Notingher, Biochim. Biophys. Acta. $2013 ; 1830,3517$.

[18]A. Ghita, F. C. Pascut, V. Sottile V and I. Notingher, Analyst $2014 ; 139,55$.

[19] B. R. Wood, S. J. Langford, B. M. Cooke, F. K. Glenister, J. Lim and D. McNaughton, FEBS Lett. 2003 ; 554, 247.

[20]S. Bonifacio, S. Finaurini, C. Krafft, S. Parapini, D. Taramelli and V. Sergo, Anal. Bioanal. Chem. $2008 ; 392,1277$.

[21]A.J. Hobro, A. Konishi, C. Coban and N. I. Smith, Analyst 2013 ; 138, 3927.

[22] K. Kong, C. J. Rowlands, H. Elsheikha and I. Notingher, Analyst $2012 ; 137,4119$.

[23] A. Naemat, H. M. Elsheikha, A. Al-Sandaqchi, K. Kong, A. Ghita and I. Notingher, Analyst $2015 ; 140,756$.

[24] A. Naemat, H. M. Elsheikha, R. A. Boitor and I. Notingher, Sci. Rep. 2016 ; 6, 20811.

[25] S. Haider, M. Wagner, M. C. Schmid, B. S. Sixt, J. G. Christian, G. Häcker, P. Pichler, K. Mechtler, A. Müller, C. Baranyi, E. R. Toenshoff, J. Montanaro and M. Horn, Mol. Microbiol. $2010 ; 77,687$.

[26] W. E. Huang, K. Stoecker, R. Griffiths, L. Newbold, H. Daims, A. S. Whiteley and M. Wagner, Environ. Microbiol. 2007 ; 9, 1878.

[27] C. Matthäus, A. Kale, T. Chernenko, V. Torchilin and M. Diem, Mol. Pharm. 2008 ; $5,287$.

[28] H. J. van Manen, A. Lenferink, and C. Otto, Anal. Chem. 2008 ; 80, 9576.

[29] C. Matthäus, C. Krafft, B. Dietzek, B. R. Brehm, S. Lorkowski and J. Popp, Anal. Chem. 2012, 84, 8549.

[30] H. N. Noothalapati Venkata and S. Shigeto, Chem. Biol. $2012 ; 19,1373$. 
[31] Y. C. Lin, E. Perevedentseva and C. L. Cheng, J. Biomed. Opt. 2015 ; $20(5), 51042$.

[32] G. Rusciano, P. Capriglione, G. Pesce, S. Del Prete S, G. Cennamo, D. Di Cave, L. Cerulli and A. Sasso, PLoS One 2013 ; 8, e72127.

[33] A. Zumbusch, G.R. Holtom, and X.S. Xie, Phys. Rev. Lett. 1999 ; 82(20), 4142.

[34] C.L. Evans, and X.S. Xie,. Annu. Rev. Anal. Chem. 2008 ; 1, 883.

[35] W. Min, C.W. Freudiger, S. Lu, and X.S. Xie, Annu. Rev. Phys. Chem. $2011 ; 62,507$.

[36] R. Mouras, P. O. Bagnaninchi, A. R. Downes and A. P. D. Elfick, J. Biomed. Opt. $2012 ; 17,116011$.

[37] T. Hellerer, C. Axäng, C. Brackmann, P. Hillertz, M. Pilon M and A. Enejder, Proc Natl Acad Sci U.S.A. 2007 ; 104, 14658.

[38] R. Mouras, P. Bagnaninchi, A. Downes, and A. Elfick, J. Raman Spectrosc. 2013 ; 44, 1373.

[39] F. De Chaumont, S. Dallongeville, N. Chenouard, N. Hervé, S. Pop, T. Provoost, V. Meas-Yedid, P. Pankajakshan, T. Lecomte, Y. Le Montagner, T. and Lagache, Nature Methods $2012 ; 9(7), 690$.

[40] N. Siamwiza, R. C. Lord, M. C. Chen, T. Takamatsu, I. Harada, H. Matsuura and T. Shimanouchi, Biochemistry $1975 ; 14,4870$.

[41] M. Hurt, S. Neelam, J. Niederkorn and H. Alizadeh, Infect. Immun. 2003 ; 71, 6243.

[42] D. R. Ledee, A. Iovieno, D. Miller, N. Mandal, M. Diaz, J. Fell, M. E. Fini and E. C. Alfonso, J. Clin. Microbiol. 2009 ; 47, 1458.

[43] W. D. Dolphin, J. Protozool. 1976 ; 23, 455.

[44] O. P. Shukla, S. M. Kaul and R. K. Mehlotra, J. Protozool. 1990 ; 37, 237-242.

[45] M. Clarke, A. J. Lohan, B. Liu, I. Lagkouvardos, S. Roy, N. Zafar, C. Bertelli, C. Schilde, A. Kianianmomeni, T. R. Bürglin, C. Frech, B. Turcotte, K. O. Kopec, J. M. Synnott, C. Choo, I. Paponov, A. Finkler, C. S. Heng Tan, A. P. Hutchins, T. Weinmeier, 
T. Rattei, J. S. Chu, G. Gimenez, M. Irimia, D. J. Rigden, D. A. Fitzpatrick, J. LorenzoMorales, A. Bateman, C. H. Chiu, P. Tang, P. Hegemann, H. Fromm, D. Raoult, G. Greub, D. Miranda-Saavedra, N. Chen, P. Nash, M. L. Ginger, M. Horn, P. Schaap, L. Caler and B. J. Loftus, Genome Biol. 2013 ; 14, R11.

[46] D. G. Dearborn and E. D. Korn, J Biol Chem. 1974 ; 249, 3342.

[47] F. R. Smith and E. D. Korn, J Lipid Res. 1968 ; 9, 405.

[48] A. G. Ulsamer, P. L. Wright, M. G. Wetzel and E. D. Korn, J Cell Biol. 1971 ; 51, 193.

[49] H. A. Saka and R. Valdivia, Annu Rev Cell Dev Biol. 2012 ; 28, 411.

\section{Figure Captions}

Fig. 1 Raman spectroscopy measurements of typical A. castellanii trophozoites after 24 hours of incubation in L-Phe(D8)-supplemented DMEM medium. (a) Brightfield image, (b) Spectral maps of selected Raman bands and (c) Selected Raman spectra at the indicated locations (i, ii, iii, iv) in the cell. Scale bar: $10 \mu \mathrm{m}$.

Fig. 2 Raman spectral imaging of $A$. castellanii at 24 hours of incubation in L-Phe(D8)supplemented media. (a) Bright field, (b) Maps corresponding to Raman spectral peaks 1449 $\mathrm{cm}^{-1}, 1004 \mathrm{~cm}^{-1}, 960 \mathrm{~cm}^{-1}$ and (c) Average Raman spectra of A. castellanii trophozoites. Scale bars: $10 \mu \mathrm{m}$. 
Fig. 3 The uptake of L-Phe(D8) by A. castellanii from ARPE-19 cells (a) Bright field image and Raman spectral maps of ARPE-19 cells infected with A. castellanii at 24 hpi and (b) Selected Raman spectra at the indicated locations. Scale bar: $10 \mu \mathrm{m}$.

Fig. 4 Raman spectral imaging of ARPE-19 cells infected with A. castellanii at 24 hpi. (a) Brightfield images and fluorescence images (recorded immediately after Raman spectroscopy measurements), (b) Maps corresponding to Raman spectral peaks $1004 \mathrm{~cm}^{-1}$ and $960 \mathrm{~cm}^{-1}$ and (c) Average Raman spectra of A. castellanii trophozoites and ARPE-19 cells. Scale bars: 10 $\mu \mathrm{m}$.

Fig. 5 Raman spectral imaging of ARPE-19 cells infected with A. castellanii at 48 hpi. Raman spectral images and selected Raman spectra of ARPE-19 cells infected with $A$. castellanii at 48 hpi. Scale bar: $10 \mu \mathrm{m}$

Fig. 6 Raman spectral imaging of ARPE-19 cells infected with A. castellanii at 48 hpi. (a) Bright field images and fluorescence images (recorded immediately after Raman spectroscopy measurements), (b) Maps corresponding to Raman spectral peaks $1004 \mathrm{~cm}^{-1}$ and $960 \mathrm{~cm}^{-1}$ and (c) Average Raman spectra from A. castellanii trophozoites and ARPE-19 cells. Scale bars: $10 \mu \mathrm{m}$.

Fig. 7 Kinetics of the uptake of L-Phe(D8) by A. castellanii from host cells. (a) Uptake of LPhe(D8) by $A$. castellanii from ARPE-19 cells: calculated ratio $\mathrm{I}_{960} /\left(\mathrm{I}_{1004}+\mathrm{I}_{960}\right)(n=6$ cells at each time point). (b) Computed difference Raman spectra for A. castellanii infecting ARPE19 cells at 24 and 48 hpi and A. castellanii cells in L-Phe(D) media at 24 hours. Spectra are shifted vertically for clarity. 
Fig 8. CARS microscopy of fixed A. castellani and ARPE-19 cells at (a) 3h, (b) 24h and (c) $48 \mathrm{~h}$ post-infection. (d) Control cells represent cell-free parasites identified at $3 \mathrm{hrs}$. Image contrast is generated from the $\mathrm{C}-\mathrm{H}_{2}$ symmetric stretching band at $2845 \mathrm{~cm}^{-1}$, which is primarily from lipid structures. The images provide complementary high-resolution threedimensional structural information generated primarily from endogenous lipid structures, allowing the interface between $A$. castellani and ARPE-19 cells to visualized. Lipid droplet structures near the interface at 48 hours. 
a

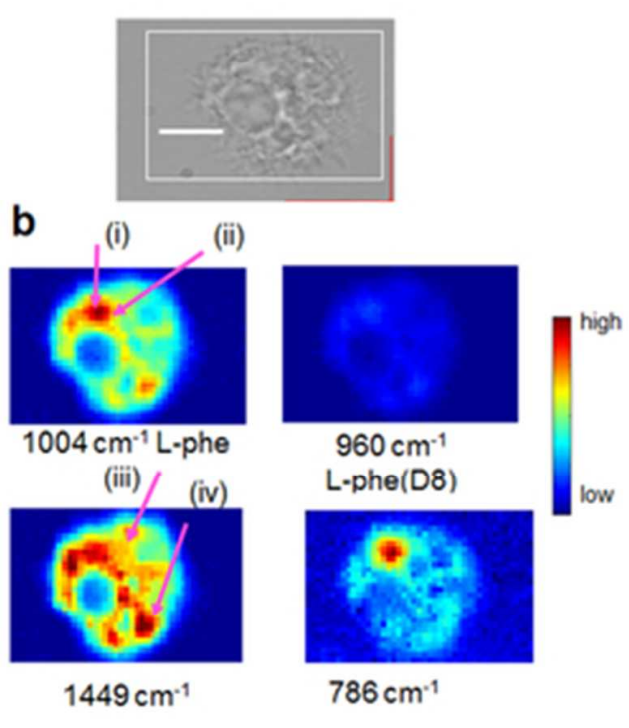

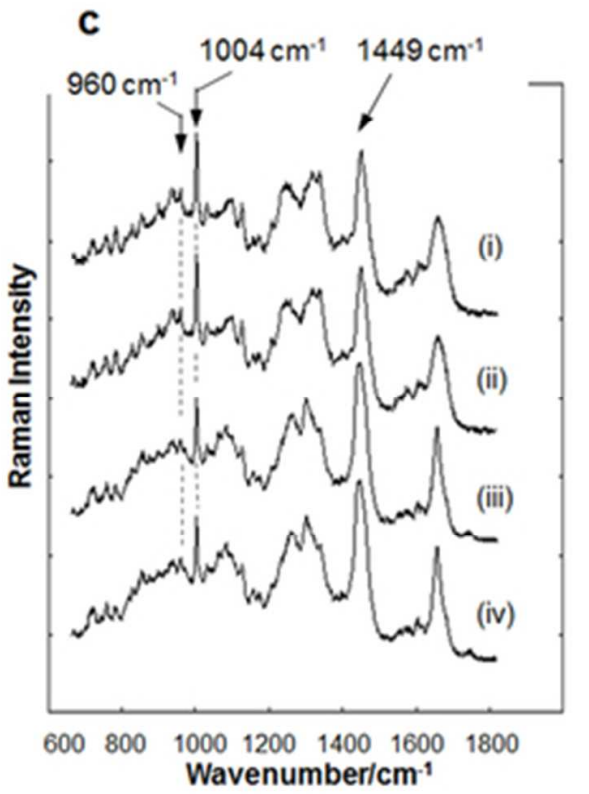

Raman spectroscopy measurements of typical A. castellanii trophozoites after 24 hours of incubation in LPhe(D8)-supplemented DMEM medium. (a) Brightfield image, (b) Spectral maps of selected Raman bands and (c) Selected Raman spectra at the indicated locations (i, ii, iii, iv) in the cell. Scale bar: $10 \mu \mathrm{m}$.

$41 \times 27 \mathrm{~mm}(300 \times 300 \mathrm{DPI})$ 
Raman spectral imaging of A. castellanii at 24 hours of incubation in L-Phe(D8)-supplemented media. (a) Bright field, (b) Maps corresponding to Raman spectral peaks $1449 \mathrm{~cm}-1,1004 \mathrm{~cm}-1,960 \mathrm{~cm}-1$ and (c) Average Raman spectra of A. castellanii trophozoites. Scale bars: $10 \mu \mathrm{m}$.

$27 \times 14 \mathrm{~mm}(300 \times 300 \mathrm{DPI})$ 


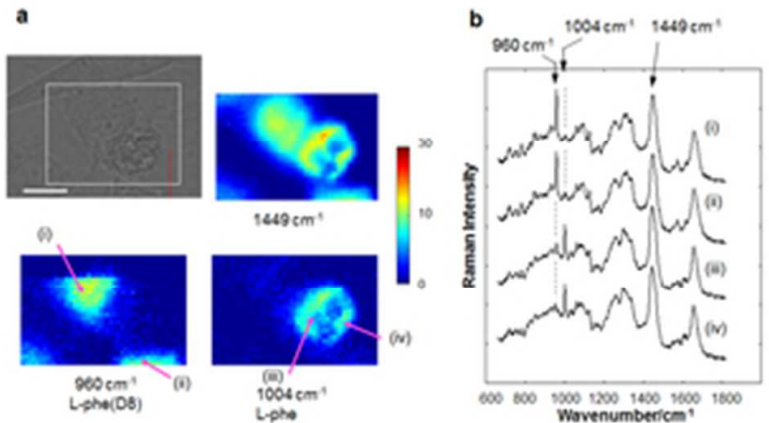

The uptake of L-Phe(D8) by A. castellanii from ARPE-19 cells (a) Bright field image and Raman spectral maps of ARPE-19 cells infected with A. castellanii at $24 \mathrm{hpi}$ and (b) Selected Raman spectra at the indicated locations. Scale bar: $10 \mu \mathrm{m}$.! +

$26 \times 14 \mathrm{~mm}(300 \times 300 \mathrm{DPI})$ 

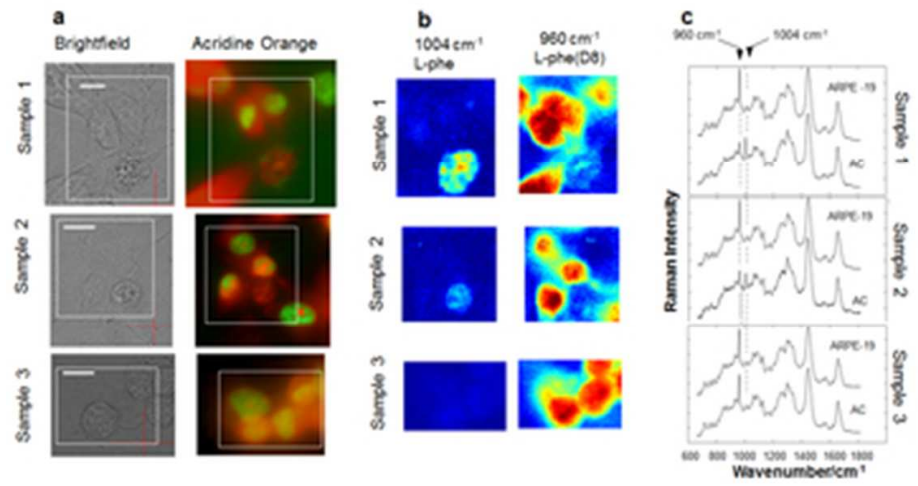

Raman spectral imaging of ARPE-19 cells infected with A. castellanii at $24 \mathrm{hpi}$. (a) Brightfield images and fluorescence images (recorded immediately after Raman spectroscopy measurements), (b) Maps corresponding to Raman spectral peaks $1004 \mathrm{~cm}-1$ and $960 \mathrm{~cm}-1$ and (c) Average Raman spectra of A. castellanii trophozoites and ARPE-19 cells. Scale bars: $10 \mu \mathrm{m}$.

$30 \times 16 \mathrm{~mm}(300 \times 300$ DPI $)$ 


\section{Raman spectral imaging of ARPE-19 cells infected with A. castellanii at $48 \mathrm{hpi}$. Raman spectral images and} selected Raman spectra of ARPE-19 cells infected with A. castellanii at $48 \mathrm{hpi}$. Scale bar: $10 \mu \mathrm{m}$

$27 \times 15 \mathrm{~mm}(300 \times 300$ DPI $)$ 
Raman spectral imaging of ARPE-19 cells infected with A. castellanii at 48 hpi. (a) Bright field images and fluorescence images (recorded immediately after Raman spectroscopy measurements), (b) Maps corresponding to Raman spectral peaks $1004 \mathrm{~cm}-1$ and $960 \mathrm{~cm}-1$ and (c) Average Raman spectra from A. castellanii trophozoites and ARPE-19 cells. Scale bars: $10 \mu \mathrm{m}$.

\section{$25 \times 13 \mathrm{~mm}(300 \times 300$ DPI $)$}



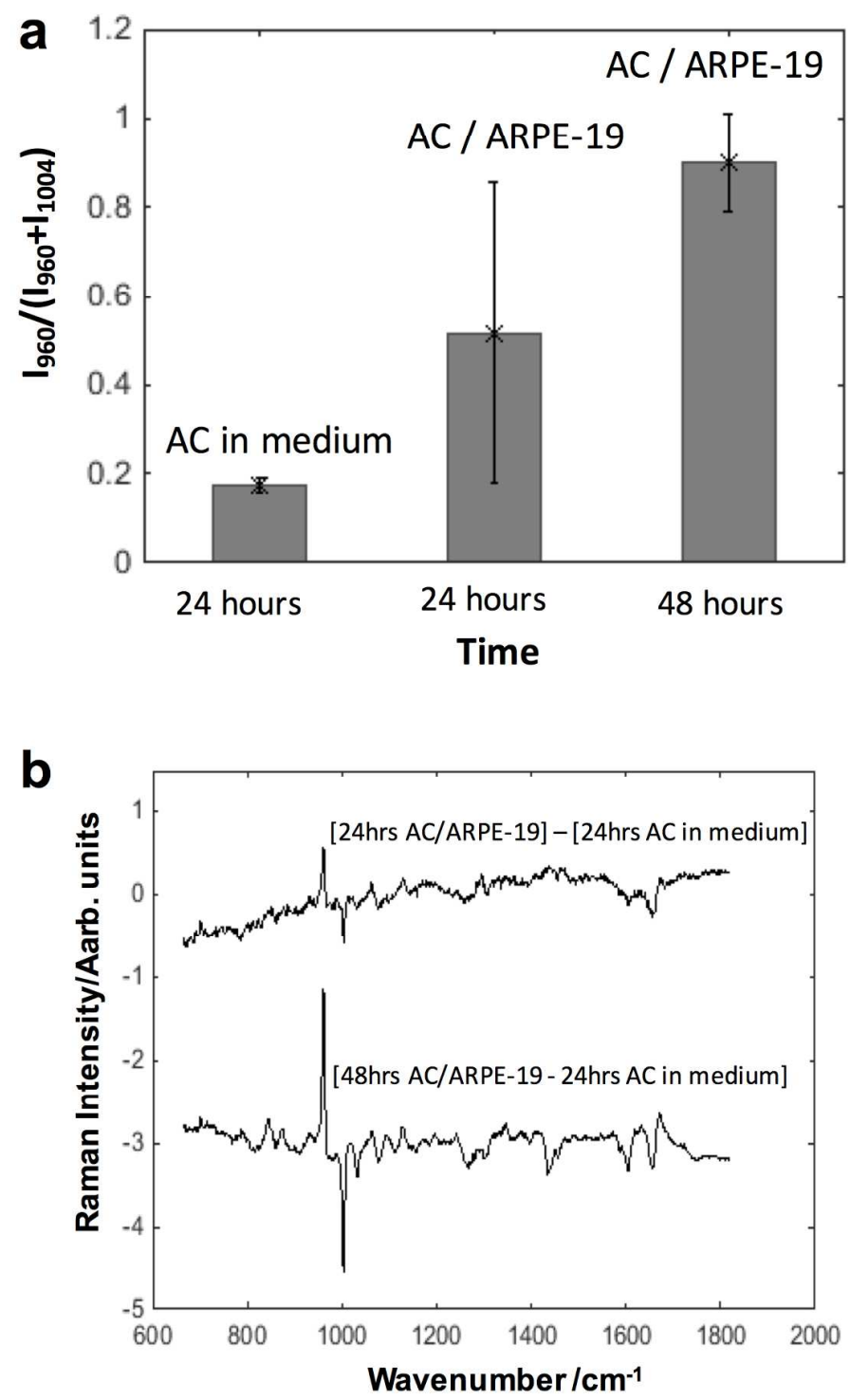

Kinetics of the uptake of L-Phe(D8) by A. castellanii from host cells. (a) Uptake of L-Phe(D8) by A. castellanii from ARPE-19 cells: calculated ratio I960/(I1004+I960) ( $n=6$ cells at each time point). (b) Computed difference Raman spectra for A. castellanii infecting ARPE-19 cells at 24 and 48 hpi and A. castellanii cells in L-Phe(D) media at 24 hours. Spectra are shifted vertically for clarity.

$$
148 \times 226 \mathrm{~mm}(300 \times 300 \mathrm{DPI})
$$


(a)
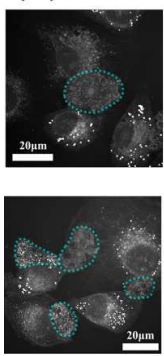

(b) 24 hours
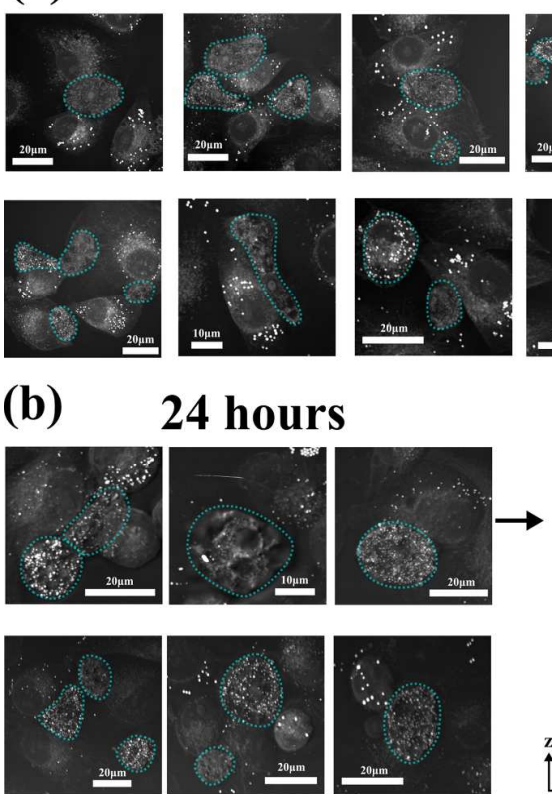

3 hours
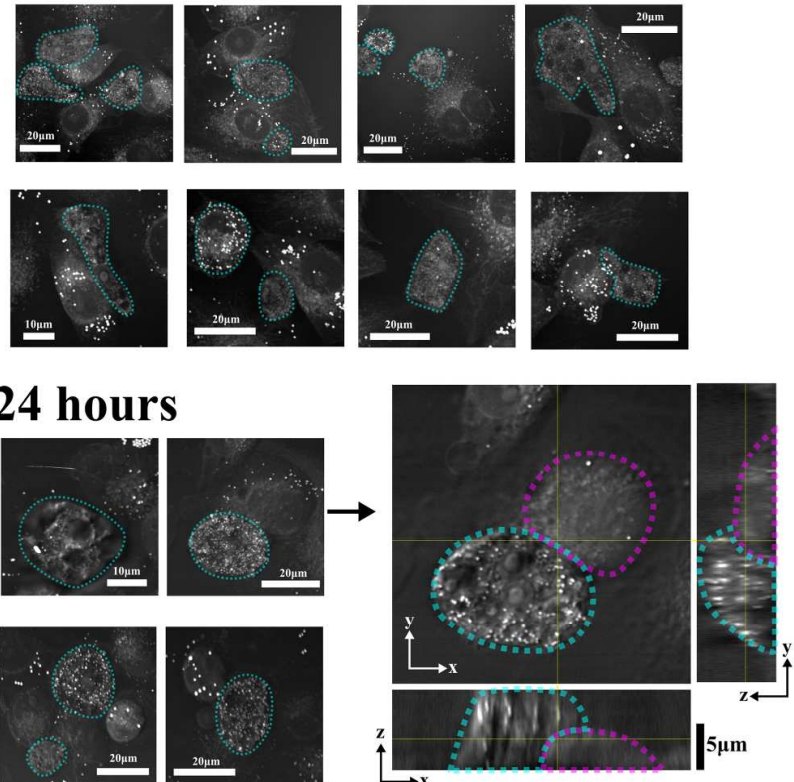

(c) 48 hours
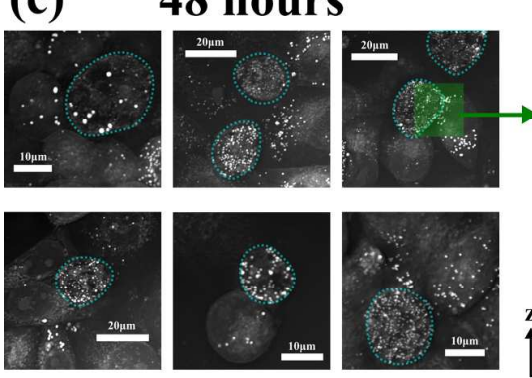

(d) Control
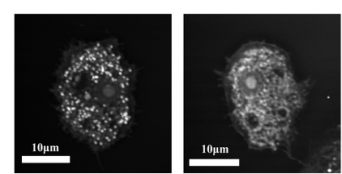

CARS microscopy of A. castellani and ARPE-19 cells at (a) $3 \mathrm{~h}$, (b) $24 \mathrm{~h}$ and (c) $48 \mathrm{~h}$ post-infection. (d) Control cells represent cell-free parasites identified at $3 \mathrm{hrs}$. Image contrast is generated from the $\mathrm{C}-\mathrm{H} 2 \mathrm{symmetric}$ stretching band at $2845 \mathrm{~cm}-1$, which is primarily from lipid structures. The images provide complementary high-resolution three-dimensional structural information generated primarily from endogenous lipid structures, allowing the interface between A. castellani and ARPE-19 cells to visualized. Lipid droplet structures near the interface at 48 hours.

$152 \times 237 \mathrm{~mm}(300 \times 300 \mathrm{DPI})$ 


\section{Page 33 of 33}

1

2

4

5

6

7

9

10

11

12

13

14

15

16

17

18

19

20

21

22

23

24

25

26

27

28

29

30

31

32

33

34

35

36

37

38

39

40

41

42

43

44

45

46

47

48

49

50

51

52

53

54

55

56

57

58

59

60
Journal of Raman Spectroscopy
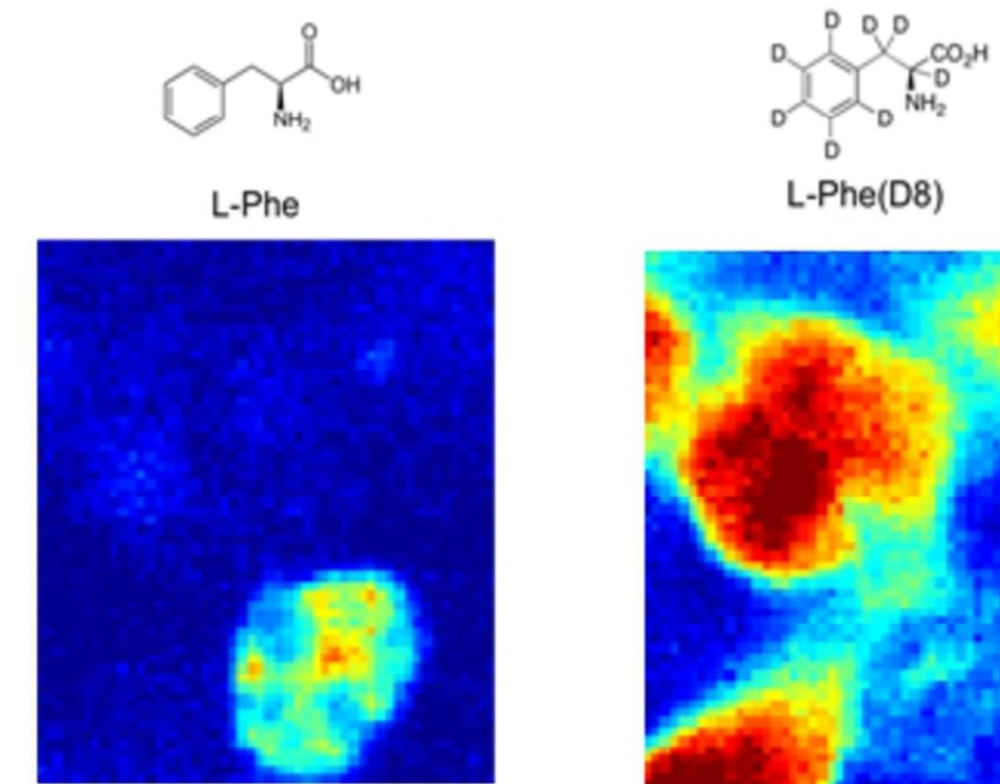

L-Phe(D8)
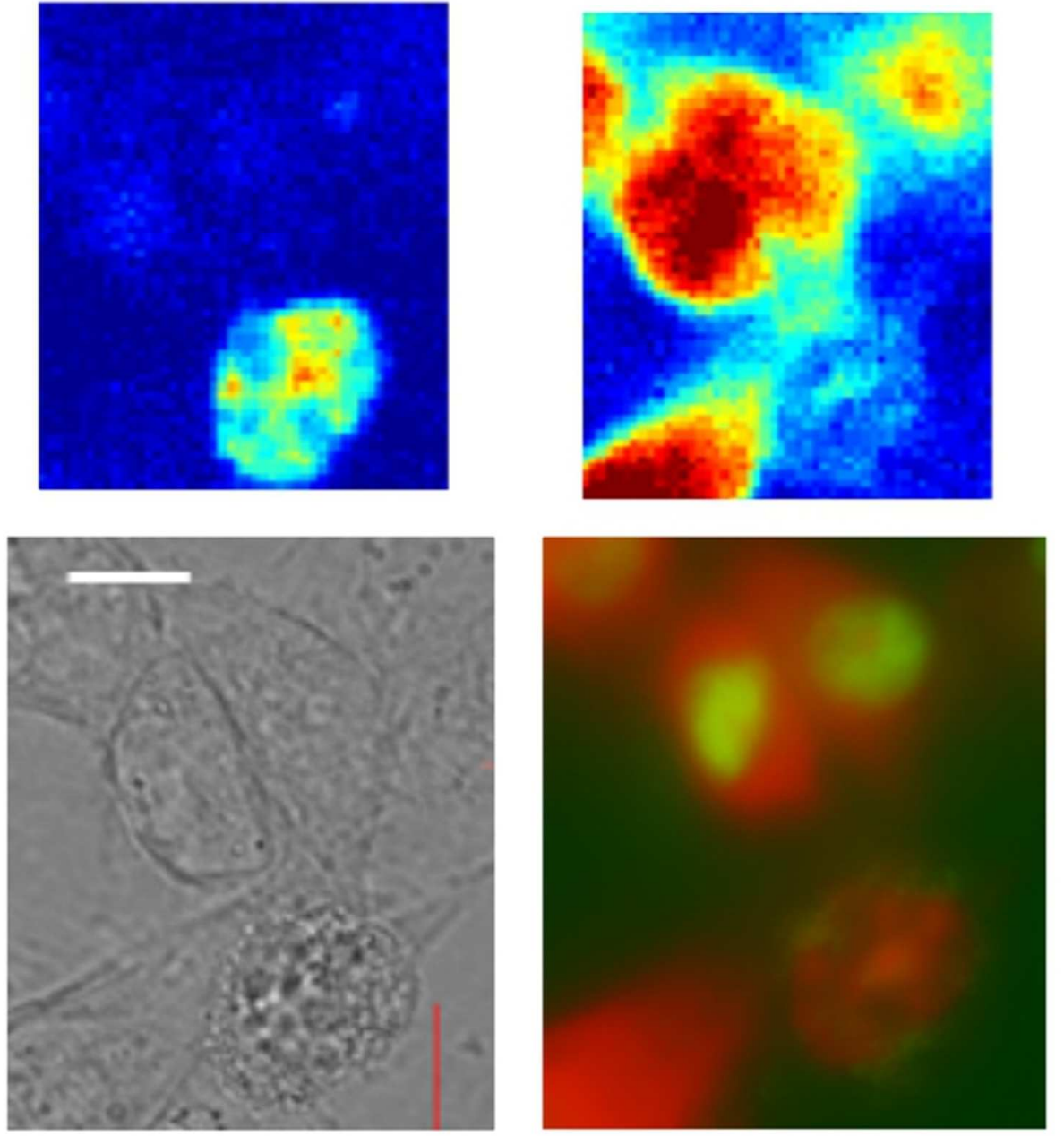

Table of Content

$59 \times 71 \mathrm{~mm}(300 \times 300 \mathrm{DPI})$

John Wiley \& Sons 